\title{
THE SCIENTIFIC RATIONALE FOR DEPLOYMENT OF A LONG-LIVED GEOPHYSICAL NETWORK ON THE MOON
}

A White Paper to be Submitted to the 2023 Planetary Science Decadal Survey

Renee C. Weber, NASA Marshall Space Flight Center, renee.c.weber@nasa.gov, 256-961-7705

Clive R. Neal, University of Notre Dame

Robert Grimm, SwRI

Matthias Grott, DLR

Nicholas Schmerr, UMD

Mark Wieczorek, OCA

James Williams, JPL/Caltech

Bruce Banerdt, JPL

Caroline Beghein, UCLA

Peter Chi, UCLA

Douglas Currie, UMD

Simone Dell'Agnello, INFN

Jared Espley, NASA GSFC

Raphael Garcia, ISAE

Ian Garrick-Bethell, UCSC

Heidi Haviland, NASA MSFC

Stephen Indyk, Honeybee Robotics

Catherine Johnson, UBC/PSI

Taichi Kawamura, IPGP

Sharon Kedar, JPL

Philippe Lognonné, IGPG

Seiichi Nagihara, Texas Tech University

Yosio Nakamura, UT Austin

Ceri Nunn, JPL

Lillian R. Ostrach, U.S. Geological Survey

Mark Panning, JPL

Noah Petro, NASA GSFC

Matthew Siegler, PSI

Thomas Watters, Smithsonian Institution

Kris Zacny, Honeybee Robotics

S. Hop Bailey, University of Arizona

Maria Banks, GSFC

Donald Barker, JSC

Hannes Bernhardt, ASU

Valentin Bickel, MPS

Josh Cahill, APL

Jackie Clark, ASU
Dani DellaGiustina, ASU

Jesse-Lee Dimech, Geoscience Australia

Andrew Dombard, UIC

Catherine Elder, JPL

Lindy Elkins-Tanton, ASU

Marshall Eubanks, Space Initiatives

Kerri Donaldson Hanna, UCF

Jan Harms, Gran Sasso Science Institute

Steve Hauck, Case Western Reserve Univ.

Lon Hood, University of Arizona

José Hurtado, UTEP

Seth Jacobson, Michigan State University

Devanshu Jha, MVJ College of Engineering

James Tuttle Keane, JPL

Amir Khan, ETHZ

Walter Kiefer, LPI/USRA

Martin Knapmeyer, DLR

Brigitte Knapmeyer-Endrun, U. of Cologne

Krishan Khurana, UCLA

Juan Lorenzo, LSU

Angela Marusiak, JPL

Patrick McGovern, LPI

Laurent Montesi, UMD

Francis Nimmo, UCSC

Deanna Phillips, UAH

Jacob Richardson, UMD

Charles Shearer, UNM/LPI

Krista Soderlund, UT Austin

Sean C. Solomon, Columbia University

Tilman Spohn, ISSI

Eleonore Stutzmann, IPGP

Sonia Tikoo, Stanford University

Slava Turyshev, JPL

Dany Waller, JHU

Ryuhei Yamada, University of Aizu

Maria Zuber, MIT 


\section{Introduction}

This white paper focuses on the scientific rationale for deploying a long-lived, global network of geophysical instruments on the surface of the Moon to understand the nature and evolution of the lunar interior. The acquired data will allow the examination of initial planetary differentiation processes that are preserved on the Moon. Evidence for such preservation comes from mare basalt samples derived from source regions consistent with having been emplaced from an early lunar magma ocean [1]. Geophysical data are critical to understanding terrestrial planet formation and early differentiation processes, and also for understanding the collision process that generated our unique Earth-Moon system. These geophysical observations of the Moon will yield a wealth of Solar System-level knowledge that builds on the Apollo geophysical experiments and exploits data from the Lunar Prospector, Kaguya, LRO, and GRAIL missions.

Over a minimum of 6 years (covering one primary tidal cycle), new data collected will characterize the nature and evolution of the lunar interior using a combination of seismic, heat flow, laser ranging, and electromagnetic sounding data. Furthermore, these data will help to constrain the Moon's current electrostatic charging environment and meteoroid impact flux, including hazard assessment. Data from a modern geophysical network will expand upon pioneering measurements made by the Apollo Lunar Surface Experiments Packages or ALSEPs (at Apollo 12, 14, 15, 16, and 17) and the two Lunokhod retroreflectors (Fig. 1). The ALSEPs returned essential data on the lunar surface environment and the lunar interior. However, fundamental questions remain unresolved, in part because of the sensitivity of the instruments but also because the ALSEP stations were clustered in a small equatorial region on the

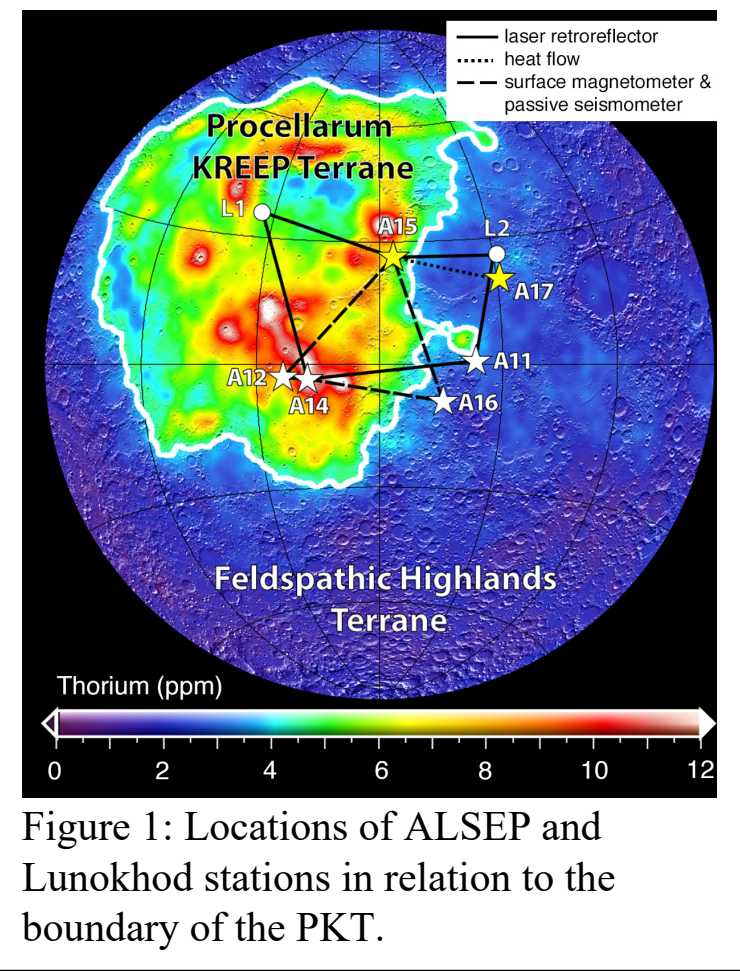
nearside of the Moon (Fig. 1). The Apollo landing sites were later discovered to straddle a geological province that differs from the rest of the Moon by its enrichment in heat producing elements, now referred to as the Procellarum KREEP Terrane (PKT) [2]. Unbeknownst to researchers at the time, much of the data acquired from the ALSEP stations are now thought to be unrepresentative of the Moon as a whole.

At the time of writing, 43 years have passed since the ALSEP stations stopped returning data from the lunar surface. In the intervening period, a wealth of observations from later orbital missions, new analyses of Apollo data and samples, and improved modeling techniques have advanced our scientific understanding of the Moon, sometimes offering conflicting hypotheses for some of the most fundamental processes that shaped the lunar interior. Taking these advances into account, it is clear that a more nuanced view of the lunar interior drives new questions that can be answered only by a Lunar Geophysical Network. 


\section{Summary of lunar internal structure}

The Moon, like other terrestrial planets, is differentiated into a crust, mantle, and core. This structure is a consequence of the accretion of the Moon from a circum-terrestrial impact-generated debris disk, and its subsequent differentiation from an initial magma ocean. To first order, the Moon's moment of inertia is roughly approximated by that of a homogeneous sphere. However, the modern Moon exhibits strong departures from the simple spherically symmetric stratified interior expected at the end of magma ocean crystallization, with hemispherical heterogeneities in crustal thickness, volcanism, magnetism, and the distribution of heat-producing elements.

Knowledge of the lunar interior stems from a long line of missions stretching back more than 50 years. Lunar Laser Ranging (LLR) has precisely monitored the Moon's solid-body motions since 1969, using retroreflector arrays deployed during Apollo and the Russian Luna missions. Changes in the round-trip laser travel time provide information on the rotation of the Moon which can be analyzed to separate orbital motion from geodynamic effects. Dissipation inferred from LLR data provided the first evidence for a fluid core, with a radius dependent upon composition.

Electromagnetic sounding of the Moon performed during and after the Apollo program provided broad constraints on core size, mantle composition, and interior temperature. Electrical conductivity and mantle temperatures were constrained at the Apollo 12 site, using concurrent surface and orbital magnetometer measurements. The observed lateral heterogeneity in electrical conductivity is consistent with the presence of the PKT. The Lunar Prospector and Kaguya magnetometers also detected an induced moment within the Moon, observed in Earth's geomagnetic tail. Under the assumption that the induced field is caused by electrical currents near the surface of a conductive metallic core, the core radius was estimated.

Seismometers were deployed on the lunar nearside at five Apollo sites, and operated continuously at four of those sites from 1969 to 1977. Many different types of naturally occurring seismic events were recorded, including deep, shallow, and thermal moonquakes, and meteorite impacts. These events continue to be analyzed to produce seismic structure models. Early models based on arrival time inversion alone were supplanted by newer models using maximum likelihood estimates, joint seismic and pre-GRAIL gravity inversion, and free oscillations. Crustal thickness estimates have decreased over the years as newer and more computationally intensive techniques were applied. The newer models mostly agree that the only major discernible discontinuity in the lunar interior is the crust-mantle boundary located at about $30 \mathrm{~km}$ depth. Although the small aperture of the Apollo passive array limited initial constraints of average radial structure below $\sim 1000 \mathrm{~km}$ depth, recent re-analyses have found evidence for a core from reflected phases.

The recent GRAIL mission mapped the Moon's gravity field in extreme detail. Shallow crustal structure is tightly constrained, but still tied to ground-truth seismic estimates at the Apollo landing sites and hence carries the associated uncertainty. Although the GRAIL mission produced a family of core models consistent with geodetic parameters and seismic constraints, perspectives differ on whether a partial melt layer in the lowermost mantle is required to satisfy available constraints. A melt layer is consistent with inversions of multiple geophysical data in combination with phaseequilibrium computations, but not required in viscoelastic dissipation models derived from laboratory measurements of deformation of melt-free polycrystalline olivine. 
Heat flow measurements were performed on Apollo 15 and 17. Characterization of the lunar global heat loss is important in understanding the thermal evolution of the Moon. In the crustmantle differentiation process, greater concentrations of radiogenic heat-producing elements (U, Th, and K) likely ended up in the crust. It is essential to quantify the crust's radiogenic contribution to the heat flow released through the lunar surface, which we are able to measure, in constraining the thermal structure of the deeper interior. Would heat production vary among the crust of different terranes or between maria and highlands? Is there any geographic variation in the heat flow out of the mantle? Such knowledge would also help us answer questions on the history of a possible lunar core dynamo, by which the Moon may have generated and maintained its own global magnetic field in the past.

Magnetism is ubiquitous in the Solar System. Both deep structure and magnetism have bearing on the now-extinct lunar dynamo, which in turn has implications for lunar thermal history and core state. Paleomagnetism and crustal magnetism studies can inform understanding of the dynamo, but the precise origin of lunar magnetic anomalies is still unclear. In addition, a magnetic low has been observed beneath the PKT, which invites questions as to the depth, history, and extent of magnetic carriers. The full nature of the extinct lunar dynamo is unknown, and even its existence on such a small body is surprising. Recent modeling shows that core convection driven by a single mechanism, in particular thermochemical convection, cannot explain either the dynamo duration or the inferred magnitude intensity of the paleofield.

The Moon's complex present-day surface record has been driven by the time-integrated history of its internal processes. The Lunar Geophysical Network mission will allow us to develop a structure model for the Moon that is consistent with all observations and provides logical context for the Moon's early history and insight into broader terrestrial planet formation and evolution.

\section{$\underline{\text { Limitations of existing data }}$}

Apollo Passive Seismic Experiment: These seismometers were deployed at every Apollo site except Apollo 17 (the instrument at Apollo 11 provided data for only 21 days) [3]. A network of four seismometers was completed in April 1972 (Fig. 1) and operated until 30 September 1977. The experiment clearly demonstrated that the Moon exhibits seismic activity at a similar level to that of an intraplate setting on Earth [4,5]. Multiple types of lunar seismic events were identified and used to infer global 1D structure [6], but lunar seismograms suffer complications compared with their terrestrial counterparts. The lunar megaregolith intensely scatters seismic energy. Secondary phases, which contain information on deep structure such as reflections of seismic energy from the crust-mantle (Moho) or core-mantle boundaries, are therefore masked by codas.

Heat Flow Experiment: Four heat flow measurements were made on the Moon during Apollo: two at the Apollo 15 site and two at Apollo 17 [7]. Unfortunately, these measurements were made in the crustal transition zones between terranes (Apollo 15) and between maria and highlands (Apollo 17), and therefore provided an ambiguous mixed signal of these geologic provinces [8,9].

Lunar Surface Magnetometers: Static magnetometers were deployed at the Apollo 12, 15 and 16 landing sites, where data were collected until 14 June 1974 [10,11]. In addition, portable magnetometers were employed as part of the Apollo 14 and 16 missions. These measurements quantified the strength and direction of the remanent crustal fields at the Apollo landing sites, and 
how they varied over kilometer length scales with the portable measurements. Apollo 15 and 16 also deployed orbiting magnetometers on subsatellites. For Apollo 12, concurrent measurements with the orbiting Explorer 35 satellite enabled probing of the lunar interior using electromagnetic induction. However, this magnetic transfer function approach was band limited by plasma effects, such that the minimum investigation depth was a few hundred kilometers. At greater depths, the temperature profiles inferred from the recovered electrical conductivity structure were in broad agreement with thermal models, but significant uncertainty remained due to a combination of measurement error and constitutive relations.

Lunar Laser Ranging (LLR) [12,13]: Retroreflectors were deployed by Apollo 11, 14, and 15 astronauts, and were also fitted to the Soviet rovers Lunokhod 1 and 2 (Luna 17 and 21 landers, respectively; Fig. 1). There are now 50 years of increasingly accurate Earth-based laser ranges. LLR analysis allows an evaluation of the deep lunar interior that extends to interactions at the coremantle boundary as well as the Moon's deepest mantle. Dissipation at the core-mantle boundary indicates the presence of a fluid core with a radius about $20 \%$ of the Moon [14,15], but an inner core has not yet been independently constrained with LLR analysis, in part because the restricted geographical extent of the existing retroreflector network limits the accuracy of lunar rotation and tide determinations [16].

\section{Unanswered questions on the lunar interior}

Despite 50 years of Apollo data analysis and more recent orbital constraints on lunar internal structure, we still do not have unambiguous observations of a mid-mantle discontinuity, the mineralogy and temperature profile of the upper mantle, the nature of the lower mantle, the presence of a partial melt layer above the outer core, or the nature of the inner core [6,17-22]. There is no consensus regarding the presence of a mid-mantle seismic discontinuity, which has been used to suggest the lower bound of an ancient lunar magma ocean (LMO). If a discontinuity is not present, or present but not global, the LMO model may need to be revised. If it is global, it suggests that the Moon did not completely melt, which has implications for its thermal evolution. Seismic data have additionally been interpreted to indicate the presence of garnet in the lower lunar mantle [23-25]. However, the same data were also interpreted to represent an increased proportion of Mg-rich olivine [18,26]. Such interpretations have implications for the bulk Moon composition.

Core constraints include [27] but do not require [28] the presence of a partial melt layer above the liquid outer core, and other analyses both support [29] and discount [30] the likelihood of its existence. The details of this deep structure are needed because they fundamentally affect the origin, extent, and duration of the lunar dynamo and the resulting record of crustal magnetic anomalies, including those at swirls. Over 50 years of laser ranging data have markedly contributed to our understanding of the lunar core and mantle, but the current network is not sufficiently distributed to provide conclusive answers regarding the size and density of the lunar core, the presence and properties of a solid inner core, and the nature of the free nutation (analogous to the Chandler wobble). Furthermore, new retroreflectors have been designed to provide more accurate ranges [31,32]; using these to expand the current network on the lunar surface would substantially improve the determination of 3D rotation and tides (and the geophysical quantities derived from them), our understanding of the deep mantle environment, and our constraints on the 
presence/absence of a solid inner core and fluid core/solid mantle boundary conditions. This expanded and enhanced LRR network will also address fundamental physics questions [33,34].

More than 7,000 deep moonquakes were recorded by Apollo, clustered in 318 source regions or nests, but $<10$ nests are undisputedly on the farside [35]. The attenuating core may prohibit detection of seismic energy from nests that could exist on the farside. Although tides are known to influence the occurrence times of deep moonquakes, the full mechanism remains unknown [36]. The precise locations and origin(s) of the rare shallow moonquakes are likewise unknown. They were initially suggested to be associated with boundaries between dissimilar surface features (e.g., impact basin rims [37]), later attributed to the interaction of the Moon with nuggets of high-energy particles ("strange quark matter") originating outside the Solar System [38,39], and most recently suggested to represent slip on tectonically active faults that underlie lobate scarps [40]. Because these are the largest lunar seismic events, they are interesting not only scientifically but also for exploration initiatives, as seismic shaking may have implications for any future infrastructure supporting a sustained human presence on the Moon.

Variations in the lunar crust (mineralogy and thickness) have been difficult to constrain far from the Apollo network sites using seismic data. Markov chain Monte Carlo algorithms have been applied to seismic wave arrival times from artificial and meteoroid impacts to estimate crustal thickness variations [41], but studies of this type are limited because the seismic arrivals from such impacts are highly uncertain. New seismic constraints are needed to provide ground truth for GRAIL's global constraints on crustal thickness [42].

Because most of the Apollo sites are located in crustal transition zones, new geophysical data from well inside areas of relatively uniform geology are needed to contextualize the two existing heat flow measurement pairs [7]. Even though we now have maps of the surface abundance of radiogenic heat-producing elements (Fig. 1), we do not know their vertical distribution through the crustal layer. Therefore, we do not have tight constraints on the total heat generated within the PKT crust as a whole and the heat production in the mantle beneath it. We also lack knowledge of the base-level heat flux outside the PKT. These represent significant knowledge gaps in defining the Moon's global heat flow budget $[8,43]$ and the bulk composition of the Moon in terms of radioactive heat-producing elements $[9,44]$.

The electrical conductivity structure of the outermost $500 \mathrm{~km}$ of the Moon, and its lateral variation, is not well understood. This zone is important as it may contain a transition from uppermantle melt residuum to pristine lower mantle, as well as differences in crustal composition and lithospheric thickness and heat flow associated with the primary geological provinces of the Moon. Similarly, the deep conductivity structure of the Moon is under-constrained [45]. A tighter average mantle conductivity profile will better constrain temperature and composition. Furthermore, very long-period measurements could distinguish a molten silicate from an iron core.

\section{Proposed instruments for a next generation geophysical network}

Four primary instruments can jointly define the interior structure of the Moon, constrain its interior and bulk composition, delineate the vertical and lateral heterogeneities within the interior as they relate to surface features and terranes, and evaluate its current seismic and tectonic activity. 
Seismometer: The recent Mars InSight mission has demonstrated that seismology continues to serve as the key tool for assessing planetary interiors. Like the SEIS package on InSight [46], a future Lunar Geophysical Network should carry a seismometer with a broad bandwidth, low noise floor, and improved sensitivity over those deployed during Apollo.

Heat flow probe: The heat flow is obtained as a product of two separate measurements of the thermal gradient and the thermal conductivity of the regolith over the depth interval penetrated by a probe [7]. The probe should reach a depth below the influence of the diurnal and annual insolation cycles $(2$ to $3 \mathrm{~m})$. Sites of measurements should be distributed among the various types and thicknesses of crust.

Magnetotelluric (MT) sounder: The MT method can greatly improve imaging of electrical conductivity structure, as it is largely insensitive to plasma effects and can achieve higher bandwidth and better depth resolution. MT measures both electric and magnetic fields and does not require a reference orbiter. Constitutive relations have been much better determined since Apollo so that, together with heat flow, composition and temperature can be robustly separated. A combination of surface measurements and orbital reference may also improve core size constraints.

Laser retroreflector: Modern retroreflectors consist of a single corner cube, eliminating temporal spreading of the laser pulse intrinsic to previous retroreflector arrays. The resulting improvements in range accuracy improve accuracy of science results by factors of 3 to more than 100 , depending upon the chosen parameter. These passive retroreflectors will have a lifetime that extends to many decades, as has been the case for the Apollo retroreflector arrays [31, 32].

Supporting measurements and technology:

1. Impact flash observation: Meteoroid impacts can be localized spatially and temporally using ground-based telescopic and orbital observations [47,48]. These impact events provide seismic sources that can be used to constrain and refine structure models.

2. Surface plasma physics: Characterization of surface plasma properties provides context for induced electromagnetic field analyses and improves understanding of the spatiotemporal input processes that influence volatile transport, surface weathering, and surface charging.

3. Farside communications relay: An optimally deployed geophysical network would be widely distributed and include one or more stations deployed on the farside. A widespread distribution would require a communications relay satellite to deliver data back to Earth.

4. Long-lived night survival and operations: To permit continuous observations, each station would require power and thermal systems capable of surviving the harsh extremes of the lunar environment, for a minimum of 6 years ( $\sim 76$ lunations).

\section{$\underline{\text { Relevance of a Lunar Geophysical Network to Solar System science }}$}

This white paper demonstrates that a globally distributed, long-lived geophysical network on the Moon, with each station containing a sensitive broadband seismometer, heat flow probe, magnetotelluric sounder, and laser retroreflector, will address many fundamental lunar science hypotheses that remain to be tested, including the magma ocean hypothesis, the stagnant lid hypothesis, and the early lunar dynamo hypothesis. Furthermore, the Moon provides a nearly pristine compositional and temporal record of formation and evolution through time, which can be 
extrapolated to model the evolution of other terrestrial planets. It is the only readily accessible body to study the relationship between parent bodies and their satellites. The volatile history of the Earth-Moon system is preserved on the Moon; electromagnetic sounding and to a lesser extent seismology can address if the lunar interior is dry and degassed, or volatile enriched. Early crustal evolution and the effects of giant impacts can inform models by which increasing fracture density enables plate recycling on larger bodies. The risk to future human exploration from moonquakes and meteoroid impacts can be addressed by long-term monitoring of the seismicity and impact rates of the Moon. Establishing a geophysical network on the Moon is therefore critical to gain a better understanding of lunar and inner Solar System science and facilitate future lunar exploration.

\section{$\underline{\text { References }}$}

[1] Taylor, S.R \& Jakes, P. (1974) Proc. Lunar Sci. Conf. 5, 1287-1305. [2] Jolliff, B. L. et al. (2000) J. Geophys. Res. 105, 4197-4216. [3] Latham, G. et al. (1969). Science 165, 241-250. [4] Nakamura, Y. (1980) Proc. Lunar Planet. Sci. Conf. 11, 1847-1853. [5] Goins, N. R. et al. (1981) J. Geophys. Res. 86, 378-388. [6] Garcia, R. F. et al. (2019) Space Sci. Rev. 215, 50. [7] Langseth, M. G. et al. (1976) Proc. Lunar Sci. Conf. 7, 3143-3171. [8] Wieczorek, M. A. \& Phillips, R. J. (2000) J. Geophys. Res. 105, 20417-20430. [9] Siegler, M. A. \& Smrekar, S. E. (2014) J. Geophys. Res. 119, 47-63. [10] Dyal, P. \& Parkin, C. W. (1972) The Moon 4, 63-87. [11] Sonett, C. P. (1982) Rev. Geophys. 20, 411-455. [12] Murphy, T. W. (2013) Rep. Prog. Phys. 76:7. [13] Müller, J. et al. (2019) J. Geod. 93, 2195-2210. [14] Williams, J. G. et al. (2006) Adv. Space Res. 37, 6771. [15] Viswanathan, V. et al. (2019) Geophys. Res. Lett. 46, 7295-7303. [16] Williams, J. et al. (2020) Planetary 2020 Decadal White Paper. [17] Lognonné, P. \& Johnson, C. (2007) in Treatise on Geophysics 69-122, Elsevier. [18] Nakamura, Y. (1983) J. Geophys. Res. 88, 677-686. [19] Khan, A. \& Mosegaard, K. (2001) Geophys. Res. Lett. 28, 1791-1794. [20] Khan, A. (2002) J. Geophys. Res. 107, No. E6:5036. [21] Lognonné, P. et al. (2003) Earth Planet. Sci. Lett. 211, $27-$ 44. [22] Khan, A. et al. (2007) Geophys. J. Int. 168, 243-258. [23] Anderson, D. L. (1975) J. Geophys. Res. 80, 1555-1557. [24] Hood, L. L. (1986) in Origin of the Moon, 361-410. [25] Hood, L. L. \& Jones, J. H. (1987) J. Geophys. Res. 92, E396-E410. [26] Nakamura, Y. et al. (1974) Geophys. Res. Lett. 1, 137-140. [27] Weber, R. C., et al. (2011) Science 331, 309-312. [28] Garcia, R. F. et al. (2011) Phys. Earth Planet. Inter. 188, 96-113. [29] Khan, A. et al. (2014) J. Geophys. Res. 119, 2197-2221. [30] Nimmo, F. et al. (2012) J. Geophys. Res. 117,E09005. [31] Currie, D. G. et al. (2013) Acta Astronautica 68, 667-680. [32] Turyshev, S. G. et al. (2013) Experim. Astron. 36, 105-135. [33] Williams, J. G. et al. (2012) Class. Quantum Grav. 29, 184004. [34] Hofmann, F. \& Müller, J. (2018) Class. Quantum Grav. 35, 035015. [35] Nakamura, Y. (2005) J. Geophys. Res. 110, E01001. [36] Weber, R. C. et al. (2009) J. Geophys. Res. 114, E05001. [37]

Nakamura, Y. et al. (1979) Proc. Lunar Planet. Sci. Conf. 10, 2299-2309. [38] Frohlich, C. \& Nakamura, Y. (2006) Icarus 185, 21-28. [39] Banerdt, W. B. et al. (2006) Adv. Space Res. 37, 1889-1893. [40] Watters, T. R. et al. (2019) Nat. Geosci. 12, 411-417. [41] Chenet, H. et al. (2006) Earth Planet. Sci. Lett. 243, 1-14. [42] Wieczorek, M. A. et al. (2013) Science 339, 671-675. [43] Laneuville, M. et al. (2018) J. Geophys. Res. 123, 3144-3166. [44] Warren, P. H. \& Rasmussen, K. L. (1987) J. Geophys. Res. 92, 3453-3465. [45] Hood, L. L. et al. (1982) J. Geophys. Res. 87, 5311-5326. [46] Lognonné, P. et al. (2019) Space Sci. Rev. 215, 12. [47] Suggs, R. M. et al. (2014) Icarus 238, 23-36. [48] Cahill, J. et al. (2020) Planetary 2020 Decadal White Paper. 